\title{
La tecnología de biomejora: ¿hasta dónde debemos garantizar las oportunidades de bienestar?
}

\author{
Bioenhancement Technology: how far we must \\ guarantee the opportunities of wellbeing?
}

OLGA CAMPOS SERENA*

\begin{abstract}
Resumen: Las biotecnologías en desarrollo tienen fuertes implicaciones más allá de los aspectos centrados en la satisfacción de ciertas necesidades individuales. Hay implicadas nuevas categorías moralmente relevantes, y tienen que ver con nuestra comprensión de la justicia distributiva, igualdad de oportunidades, nuestras obligaciones y derechos como padres, y nuestras obligaciones morales hacia los animales no humanos. Esta última cuestión es considerada particularmente determinante si demostramos que ellos son sujetos de derechos morales. Cuando intentamos determinar las prescripciones prácticas de este esquema argumentativo nos encontraremos con la obligación de analizar si las técnicas de mejora podrían ser consideradas como una herramienta para proporcionar más igualdad, dadas las situaciones naturales individuales de partida tan diferentes.

Palabras clave: Ética de la mejora, animales no humanos, igualdad de oportunidades.
\end{abstract}

\begin{abstract}
The biotechnologies in developing have strong implications beyond the aspects focused on satisfying certain individual needs. There are new and morally relevant categories involved, and they have to do with our understanding of distributive justice, equality of opportunities, our obligations as well as rights as parents, and our moral obligations towards nonhuman animals. This last question is considered particularly determinant if we demostrate that they are subjects of moral rights. When we try determinate the practical prescriptions of this argumentative scheme we will find us with the obligation to analyze if enhancement techniques could be conceived as a tool to provide more equality, given that naturally individual situations are so different at the outset. Key words: Ethics of enhancement, nonhuman animals, equality of opportunities.
\end{abstract}

Este texto es una primera aproximación a la cuestión de hasta dónde se generan obligaciones morales hacia los agentes una vez reconocemos la plausibilidad de una propuesta teórica que reconozca derechos morales para los animales. Reivindicar la necesidad de ampliar

Fecha de recepción: 03/06/2016. Fecha de aceptación: 25/07/2016.

* Universidad de Granada, Profesora Colaboradora, olgacampos@ugr.es. Líneas de investigación: Ética Práctica, Derechos Morales de los Animales, Ética de la Mejora. Publicaciones recientes: LARA, F. Y CAMPOS, O. (2015), Sufre, luego importa. Reflexiones éticas sobre los animales, Madrid, Plaza y Valdés. CAMPOS SERENA, Olga (2016), The ethics of enhancement: cognitive inequalities and sentient nimals, Ramon Llull Journal of Applied Ethics. 7, pp. 71 - 91. 
la comunidad moral hasta abarcar a individuos no humanos es algo que, obviamente, tendrá que justificarse. El escollo, no obstante, no tendría tanto que ver con dicha justificación en sí misma como con las implicaciones prácticas que podría generar. En concreto, aquí me he interesado por una de ellas que tiene que ver con los nuevos desarrollos biotecnológicos. La conocidad como ética de la mejora reflexiona sobre las razones a favor y en contra de la mejora de individuos a través de una tecnología novedosa que posibilita modificaciones que van más allá de los clásicos usos terapeúticos.

El asunto se concreta en las nuevas posibilidades a las que hay que hacer referencia. Suele hablarse de la altura o la inteligencia, pero ¿y si pudiera hacerse a las personas más felices desde un punto de vista biológico? Y dado que estamos ante un concepto definitivamente ambiguo, ¿habría algunas características que pudiéramos relacionar con ella? El problema no es sencillo, pero sí es fácil ver que previamente estaría en juego en exhaustivo análisis ético de las tecnologías de mejora. Las claves irían desde lograr identificar las dificultades teórico-prácticas que definen este debate hasta contar con que entrarían en escena importantes cuestiones relacionadas tanto con nuestra propia identidad humana como con las radicales consecuencias sociales y políticas previsibles. En los últimos años encontramos autores muy destacados, como Julian Savulescu, encargándose de encarar el desafío y escribiendo o editando volúmenes que recogen las discusiones más interesantes al respecto (Savulescu, \& Bostrom, 2009) (Savulescu, ter Meulen, \& Kahane, 2011). Las respuestas críticas también son representativas. Las malas e imprevisibles consecuencias o el cuestionamiento de la naturaleza humana son argumentos habituales (Sandel, 2004, 2007), pero también cuestionables (Kamm, 2005, 2009). Una de las contrarréplicas más esgrimidas apunta a la posibilidad de anticipar las consecuencias desigualitarias investigando las formas más adecuadas de gestionar globalmente esta nueva tecnología (Buchanan, 2011). Otra señala que hay un tipo de mejora que se libraría de las críticas en torno a las malas consecuencias sociales de este tipo de aplicaciones biotecnológicas. Se trata de la mejora moral de individuos y sus consiguientes buenas implicaciones desde el punto de vista consecuencial. La discusión que surge al respecto es amplia e interesante (Persson, \& Savulescu, 2012; Campos, 2010; Douglas, 2008; Faust, 2008).

De forma que el componente ético de la evaluación ha de traspasar, si se quiere ser riguroso, el enfoque individual. Hay que poner el acento en el hecho de que ya no se trata de pensar sobre las implicaciones de mejoras individuales concretas sino que el verdadero interés filosófico recae en el contexto de lo público. La posibilidad de satisfacer necesidades individuales llevará necesariamente unidas categorías muy distintas de los propios intereses particulares y con un elemento social en común. Estará ligada nuestra comprensión de la justicia distributiva y nuestro nivel de compromiso con la igualdad de oportunidades. Igualmente, si estamos preguntando por la legitimidad de una sociedad con futuros individuos mejorados entonces el papel de la maternnidad/paternidad será completamente relevante. Identificar nuestras obligaciones y derechos como padres será algo especialmente relevante (Savulescu, 2001, 2007).

$\mathrm{Y}$, en este contexto en el que se está abriendo un campo enorme de posibilidades en torno a las mejoras individuales y sociales, ¿no tendríamos también algo que decir en torno a nuestras obligaciones morales hacia las personas con discapacidad? La respuesta que se defiende en esta presentación es una afirmación. Una afirmación que estará directamente 
relacionada con el tipo concreto de mejoras que reconozco como especialmente pertinentes. He identificado la posibilidad de lograr que las personas sean más felices como una de las posibilidades más relevantes entre todas las que ofrecen las nuevas tecnologías de biomejora. Al mismo tiempo he reconocido la ambigüedad de la pretensión por la ambigüedad misma del concepto. Consideremos sin embargo la posiblidad de simplificar el asunto preocupándonos por mejorar, no tanto la felicidad misma sino las oportunidades de ser feliz (entendidas como oportunidades de bienestar). Ahora bien, ¿tener más oportunidades, en este sentido, podría estar relacionado con el abanico de capacidades del que cada uno disponemos? Si así fuera no sería complicado reconocer la estrecha relación que podría darse entre las posibilidades de mejoras individuales que promete la nueva biotecnología y el potencial igualador que éstas introducen. Así, si dispusiéramos de la tecnología necesaria para asegurar que los miembros de la comunidad moral tuvieran el tipo de capacidades en juego para disponer de un abanico suficientemente amplio de oportunidades de bienestar, ¿acaso no deberíamos entonces promoverla desde un punto de vista ético? De lo que no cabe duda es de que la propia naturaleza genera situaciones individuales de partida tan desiguales que, si las técnicas de mejora resultan ser una buena herramienta para introducir más igualdad, no debería obviarse la posibilidad de corregir algunas desventajas en origen. Se trataría de extender nuestra idea de igualdad a un contexto previo, pero con el mismo objetivo: asegurar el mismo acceso a determinados bienes. Lo que caiga dentro del contenido de los citados bienes seguramente dará lugar a una interesante discusión. Lo que aquí porpongo es centrarnos en aquellos bienes que, de alguna forma, podrían estar objetivamente relacionados con la oportunidad de aumentar nuestro bienestar individual.

Aclaremos la relación postulada entre las prácticas habitualmente conocidas como enhancement y el potencial igualatorio de las mismas. Es muy útil ilustrar la apuntada conexión mediante un ejemplo bien conocido. Prestemos atención a las prácticas de dopaje en el deporte de competición. La postura habitual es tomar partido a favor del fair play, es decir, cualquier práctica que implique una modificación artificial de la situación de partida del deportista será considerada morlamente errónea. Lo interesante es pensar en cómo realmente tendríamos que valorar el hecho de que se den situaciones de partida tan diferentes. No debería obviarse que, si queremos evitar la arbitrariedad y fomentar una competición justa, entonces puede que una buena forma de hacerlo sea controlando y permitiendo el dopaje. Podría tratarse de sustancias hasta ahora ilegales o, incluso, de llevar a cabo modificaciones sustanciales en el ámbito de la configuración genética. Esta sería una buena forma de asegurar la igualdad de oportunidades en el deporte de competición. Sólo así tiene sentido dar verdadera importancia al nivel de dedicación o disciplina, sabiendo que los resultados deportivos individuales obedecen al esfuerzo personal y no al arbitrario mecanismo de la lotería natural (Pérez Triviño, 2011, 2013).

¿Tiene sentido esgrimir un argumento similar con respecto a las oportunidades de bienestar? Es decir, imaginemos un escenario en el que podemos contar con la posibilidad de asegurar las mismas oportunidades de satisfacción individual mediante las tecnologías nanobio-info-cognitivas: ¿tendríamos que considerar seriamente ésta como una de las cuestiones más urgentes de la ética práctica? Indudablemente las preguntas al respecto ni serían pocas ni fáciles de resolver. Para empezar surge la duda en torno al compromiso con una determinada comprensión del bienestar, sin embargo este análisis no lleva implícita una concepción uni- 
versal de bienestar. Para evitarlo desde el principio se ha hablado de oportunidades como la manera de asegurar distintas opciones de satisfacción, y entendiendo tal libertad de elección como un bien primario en sí mismo compatible con los dintintos planes de vida posibles.

La controversia no sólo se sitúa aquí sino también en la asunción de que hay ciertas capacidades cognitivas estrechamente relacionadas con el apuntado abanico de oportunidades. La existencia de una conexión entre las posibilidades individuales de experimentar bienestar y la sofisticación psicológica ha sido muy considerada históricamente, pero ello no la hace menos controvertida. El esquema teórico en el que se enmarca esta presentación entronca con un enfoque bienestarista desde el que la búsqueda de la mejor vida posible (para cualquier sujeto de derechos morales) se convierte en el objetivo promordial del buen uso de la biotecnología. En dicho contexto, cuando se habla de oportunidades de bienestar no se estaría tratando de identificar los bienes intrínsecos específicos experimentados en un momento dado, sino que se estaría poniendo el énfasis en las capacidades individuales particulares para el bienestar. Está presente el análisis de J. S. Mill cuando se afirma que mayor capacidad cognitiva supone abrir el campo de posibilidades en torno al bienestar, y que cuanto mayor y más variado sea el señalado abanico de oportunidades, más posibilidad de obtener cosas valiosas que van más allá del placer más básico. Ello tendrá un valor objetivo que, en opinión de Mill, habrá de estar suficientemente equilibrado con las justas demandas de un punto de vista más subjetivista. El autor lo resuelve desde su teoría del placer y postulando la figura de los jueces competentes. Existen cierto tipo de sensaciones placenteras cualitativamente superiores a las provenientes de satisfacer necesidades físicas o deseos poco sofisticados. Pero, ¿cómo se justificada la señalada superioridad? Por las preferencias subjetivas individuales de aquellos que pueden experimentar todo el espectro de experiencia de bienestar. (Mill, 1863).

La cuestión se presenta con especial pertinencia si asumiéramos que es posible contar con la tecnología necesaria para mejorar a los individuos en línea con la supuesta superioridad que Mill reivindica. Habría que entenderlo reconociendo en las mejoras un carácter instrumental respecto a ciertos bienes objetivos. En terminología rawlsiana, estaríamos incrementado los bienes primarios, esto es, estaríamos asegurando el ejercicio de los derechos básicos o libertades, relacionados con ciertos bienes generales que cualquier agente racional consideraría deseables, con independencia de su concepción de bien y de su plan de vida (Allhoff, 2005). Si la mejora cognitiva pudiera entenderse como una forma legítima de corregir una situación desventajosa de partida, entonces caería dentro de esta categoría. ¿Acaso no podríamos considerar un bien objetivo ampliar las oportunidades de satisfacción de los individuos con graves discapacidades cognitivas, si admitiéramos la relación entre ambas cosas? Si así fuera la siguiente pregunta es obligada, ¿estaríamos legitimados o acaso obligados a hacerlo? Aquellos que reivindican la superación de enfoques medicalizados y paternalistas de la discapacidad tendrán mucho que decir al respecto (Seoane, 2011). La propuesta de la diversidad funcional (Barnes, \& Mercer, 2010), que apuesta por comprender el fenómeno desde el reconocimiento de formas distintas de estar en el mundo, probablemente defenderá que algo falla si contestamos afirmativamente a la pregunta. Los defensores de la mejora cognitiva, por su parte, afirmarán que el hecho de que algunos individuos con graves discapacidades no tengan acceso a determinadas formas complejas de bienestar podría interpretarse como una discriminación injustificable, si contáramos con la posibilidad de llevar 
a cabo la mejora y no la lleváramos a cabo. En esta línea podríamos pensar que, en algún sentido, estamos dañando a alguien privándolo, por ejemplo, de la posibilidad de relacionarse con los demás en un sentido pleno o de poder auto-descubrir sus propias capacidades y características individuales.

Por si fuera poco el asunto se complica si estamos comprometidos con un paradigma ético no antropocéntrico. Si reconocemos la coherencia de superar el paradigma racionalista clásico y la plausibilidad de sustituirlo por otro que tenga en cuenta la capacidad de sufrir de los individuos sin importar su especie, entonces nos encontraremos con nuevos miembros de la comunidad moral para los que igualmente podría plantearse la cuestión del enhancement. Reivindicar el paradigma animalista podría llevar a considerarlos miembros plenos de la comunidad moral, y así generar una fuerte responsabilidad moral de los agentes morales hacia ellos. Los criterios racionales y no arbitrarios deberían guiar nuestras relaciones con ellos (Singer, 1975). Ello hace que no sea irrelevante la pregunta acerca de si las demandas de igualdad también exigirían una mejora cognitiva en su caso (Lara, \& Campos, 2015). La primera impresión que probablemente tendremos es la de que una prescripción moral de ese tipo en su caso resulta muy contraintuitiva. Obviamente, si estamos interesados en mostrar que tal obligación no tiene sentido tendríamos que aportar otras razones.

El debate en torno a los derechos morales de los animales ha dado lugar a una amplia discusión teórica y práctica en las últimas décadas. Los nuevos desarrollos biotecnológicos hacen que resurja la discusión, dada las implicaciones tan drásticas que éstas podrían tener en el contexto de la concreción de tales derechos individuales. No es difícil demostrar la necesidad de superar un paradigma ético anacrónico, donde está fuertemente instalado un prejuicio especista que lleva a discriminar arbitrariamente a todo el que no caiga en la categoría de homo sapiens (Campos, 2011). Intentar solucionar el problema de la arbitrariedad con la propuesta de la racionalidad moral como criterio de considerabilidad moral tampoco da buenos resultados. No es el lugar de entrar en el contenido del debate pero sepamos que las incoherencias del antropocentrismo ético no son pocas ni carecen de importancia. Además de dejar fuera a un grupo de humanos (como los niños pequeños, por ejemplo) incapaces de satisfacer el criterio (tendrán derechos morales por clausulas ad hoc), está el problema de la elección misma del criterio (Dombrowski, 1997). ¿Por qué la racionalidad moral debe ser lo que ha de determinar la protección moral? Si se trata de proteger a los individuos de posibles daños infligidos por otros, ¿entonces por qué no tener en cuenta la capacidad de sufrir en lugar de la capacidad de entender los derechos de los demás? (Holtug, 2007). Los animales no humanos son mucho más inteligentes de lo que históricamente se ha asumido (Searle, 1994), pero no tanto como para satisfacer el criterio antropocéntrico de la reciprocidad moral tal como éste se ha entendido en nuestra tradición ética. Tiene más sentido (muchos lo llaman relevancia contextual), si estamos intentando determinar quiénes han de contar con protección moral, preguntar si alguien puede sufrir o no. Difícilmente se puede dudar de la pertinencia de atribuir estados mentales desagradables a muchos animales no humanos, no obstante, la herencia mecanicista cartesiana todavía hoy sigue haciendo necesario explicitar los argumentos (Perrett, 1997).

$\mathrm{Si}$, a pesar de las reservas, consideráramos legítima la premisa de que los individuos con una discapacidad cognitiva sufrirán algún tipo de daño extrínseco si tenemos la posibilidad aumentar sus oportunidades de bienestar y no lo hacemos, entonces esto tendría importantes 
implicaciones éticas para los animales no humanos que estén dentro de la comunidad moral. Así, podría entenderse que dentro del conjunto de los menos afortunados (aceptada una relación entre ciertos niveles de límite de cognición y oportunidades de bienestar) no sólo estarán aquellos con fuertes discapacidades cognitivas sino también los no humanos sensibles. La idea es que en ambos casos tendrían que activarse ciertos mecanismos compensatorios, que estarían por determinar pero que serían necesarios desde el punto de vista de la justicia.

La radical importancia ética de las nuevas biotecnologías de mejora radica precisamente en este punto. ¿Cómo podríamos obviar la posibilidad de aumentar el abanico de este tipo de oportunidades? ¿Cómo cuestionar este objetivo desde el punto de vista de la justicia y en tanto condición de posibilidad de otras cosas valiosas? Nuestras intuiciones morales probablemente buscarán la forma de hacerlo. Es difícil estar de acuerdo a priori con tan radical cambio, especialmente en el caso de los animales. Pero, como filósofos, deberíamos estar dispuestos a buscar razones que sirvan para justificar nuestra posición intuitiva. Tratar el asunto de forma racional exige poner en cuestión los prejuicios instalados en la evaluación de cualquier nueva tecnología. No obstante, aquí no sólo están en juego las razones que aportan los transhumanistas a favor de las mejoras sino también importantes cuestiones de justicia distributiva sobre las que tendríamos que ponernos de acuerdo. De lo que no cabe duda es de que, incluso muchos de aquellos que no son bioconservadores, marcan una línea con la que pretenden demarcar las situaciones en las que compensaríamos a los humanos que han tenido poca suerte en la lotería natural del caso de los animales (donde, afirman, no se activarían los mecanismos compensatorios). La razón que suelen esgrimir para justificar la diferencia entre unos y otros no suele descansar en negar el status moral de los segundos sino, más bien, en un enfoque desde el que se quiere reivindicar una diferencia moralmente relevante entre ambos grupos de individuos apelando a "lo que es natural" en cada caso. Inspirados, en muchos casos, en análisis como el enfoque de las capacidades de M. Nussbaum, defienden la plausibilidad de limitar la obligación de mejora cognitiva sólo a los humanos apelando al conjunto de capacidades que los caracterizan como especie. Cuando habla de florecimiento hace referencia al adecuado desarrollo de las apuntadas capacidades. Así, con relación a aquellos que tienen una grave discapacidad cognitiva demanda la obligación de generar escenarios donde el desarrollo de su potencial pueda alcanzar los mismos niveles de oportunidades que los demás (Nussbaum, 2006, 2012). Estaríamos fallando en nuestra obligación de proporcionar una igualdad de oportunidades en torno al propio florecimiento si, como sucedería al obviar la potencialidad de la biotecnología, no invertimos en las herramientas que permiten un adecuado desarrollo de las capacidades naturales. Capacidades éstas que se medirían atendiendo a aquello que es normal para nuestra especie. Por tanto, advertirían, la prescripción de mejora cognitiva se sostiene para los humanos con capacidades cognitivas por debajo de la norma pero no para los animales no humanos.

La pretensión es evitar la posible obligatoriedad en el segundo caso insistiendo en que sus capacidades no tienen que ser normalizadas. Las oportunidades para su florecimiento pueden asegurarse sin necesidad de recurrir a aumentar su nivel cognitivo, puesto que el desarrollo de sus capacidades naturales (no mermadas en su caso) no lo requiere. Sin embargo, aunque asumir este enfoque nos libraría de muchas consecuencias contraintuitivas, no podemos pasar por alto la arbitrariedad de sus presupuestos. Lo que encontramos debajo de este tipo de argumento es el conocido como Species-Norm Account. Apelar a lo que es normal para 
una especie con el objetivo de determinar los derechos morales de la misma, además de arbitrario es especista (McMahan, 1996, 2002). La irrelevancia moral de este criterio debería hacer que no estuviéramos dispuestos a aceptar que lo que se considera normal para cada especie sea lo único determinante en el establecimiento de los principios de justicia.

El análisis ético que se demanda es radicalmente complejo, en parte porque todavía estamos en un escenario posible con respecto a las nuevas biotecnologías de mejora. Pero, lejos de ser ésta una razón para ilegitimar la discusión, debería ser un estímulo para ella. El asunto de hasta dónde estarán justificadas las prescripciones morales en torno a la biomejora cognitiva no está resuelto. Lo que sí sabemos es que el papel que al respecto han de jugar los animales no humanos en nuestras consideraciones de justicia no es moralmente irrelevante. Y también que el problema de que puedan sufrir algún tipo de daño extrínseco, si podemos aumentar sus oportunidades de satisfacción y no lo hacemos, no puede resolverse apelando a la escasa potencialidad de sus capacidades naturales. Ello no implica que tengamos que obviar los enormes problemas de plausibilidad en juego. Las complicaciones teóricas y prácticas son casi inabarcables, desde las consecuencias completamente imprevisibles hasta la ingente cantidad de recursos que demanda. Lo que sí implica es la necesidad de hacernos cargo de una dicotomía real, que la ética no puede pasar por alto: o bien abandonamos la pretensión de proporcionar el escenario más justo para los miembros de la comunidad moral, o bien encontramos una buena forma de cuestionar la extensión de la obligación de mejora a los animales.

\section{Bibliografía}

Allhoff, F. (2005): Germ-line genetic enhancement and Rawlsian primary goods, Kennedy Institute of Ethics Journal, $\mathrm{n}^{\mathrm{o}} 15$ (1).

Barnes, C. \& Mercer, G. (2010): Exploring disability (3 ${ }^{\text {rd }}$ edition), Cambridge, Polity.

Buchanan, A. (2011): Beyond humanity?: the ethics of biomedical enhancement, Oxford, Oxford University Press.

Campos Serena, O. (2010): La mejora del carácter moral en la evaluación de las técnicas de mejora biológica, Dilemata. Revista Internacional de Éticas Aplicadas, nº 3 .

Campos Serena, O. (2011): Más allá de una concepción instrumental del valor de los animales: la irracionalidad del paradigma humanista, Revista de Filosofía, no 36.

Dombrowski, D.A. (1997): Babies and Beasts, Campaign, University of Illinois Press.

Douglas, T. (2008): Moral enhancement, Journal of Applied Philosophy, $\mathrm{n}^{\circ} 25$ (3).

Faust, H. S. (2008): Should we select for genetic moral enhancement? A thought experiment using the Moralkinder (MK+) haplotype, Theoretical Medicine and Bioethics, $\mathrm{n}^{\mathrm{o}} 29$.

Holtug, N. (2007): Equality for animals, en Ryberg, J., Petersen, T. \& Wolf, C. (Eds.), New waves in applied ethics, Basingstock, Palgrave Macmillan.

Kamm, F. M. (2005): Is there a problem with enhancement?, American Journal of Bioethics, $\mathrm{n}^{\mathrm{o}} 5(3)$.

Kamm, F. M. (2009): What is and is not wrong with enhancement?, en Savulescu, J. \& Bostrom, N. (Eds.), Human enhancement, Oxford, Oxford University Press.

Lara, F. y Campos, O. (2015): Sufre, luego importa. Reflexiones éticas sobre los animales, Madrid, Plaza y Valdés. 
McMahan, J. (1996): Cognitive Disability, Misfortune, and Justice, Philosophy \& Public Affairs, $\mathrm{n}^{\mathrm{o}} 25(1)$.

McMahan, J. (2002): The ethics of killing: problems at the margins of life, Oxford, Oxford University Press.

Mill, J. S. (1863): Utilitarianism, Oxford, Oxford University Press, 1871, 1998.

Nussbaum, M. (2006): Las fronteras de la justicia. Consideraciones sobre la exclusión, Barcelona, Paidós, 2007.

Nussbaum, M. (2012): Crear capacidades. Propuesta para el desarrollo humano, Barcelona, Espasa.

Pérez Triviño, J. L. (2011): Ética y deporte, Bilbao, Desclée de Brouwer.

Pérez Triviño, J. L. (2013): The challenges of modern sport to ethics: from doping to cyborgs, Lanham, Lexingnton Books.

Perrett, R. (1997): The analogical argument for animal pain, Journal of Applied Philosophy, $\mathrm{n}^{\mathrm{o}} 14$.

Persson, I. \& Savulescu, J. (2012): Unfit for the future: the need for moral enhancement, Oxford, Oxford University Press.

Sandel, M. (2004): The case against perfection, Atlantic Monthly, no 293 (3).

Sandel, M. (2007): Contra la perfección. La ética en la era de la ingeniería genética, Barcelona, Marbot.

Savulescu, J. (2001): Procreative Beneficence: why we should select the best children, Bioethics, $\mathrm{n}^{\mathrm{o}} 15$.

Savulescu, J. (2007): In defence of procreative beneficence, Journal of Medical Ethics, $\mathrm{n}^{\circ}$ 33.

Savulescu, J. \& Bostrom, N. (Eds.) (2009): Human enhancement, Oxford, Oxford University Press.

Savulescu, J., Ter Meulen, R. \& Kahane, G. (Eds.) (2011): Enhancing human capacities, Oxford, Wiley-Blackwell.

Searle, J. (1994): Animal minds, en French, P. A., Uehling, T. E. y Wettstein H. K., J. R. (eds.) (1994), Midwest studies in philosophy XIX: philosophical naturalism, Notre Dame, University of Notre Dame Press.

Seoane, J. A. (2011): ¿Qué es una persona con discapacidad?, Ágora. Papeles de Filosofía, $n^{\circ} 30$ (1).

Singer, P. (1975): Liberación animal, Madrid, Trotta, 1999. 